\title{
Evaluación de la aplicabilidad de especies forestales de la serranía peruana en fitorremediación de relaves mineros
}

\section{Assessment of the applicability of phytoremediation in mining tailings using forest species of the peruvian highland}

\author{
Javier Paredes Mur \\ Universidad Católica de Santa María; Urb. San José s/n, Yanahuara, Arequipa
}

DOI: https://doi.org/10.33017/RevECIPeru2014.0013/

\section{Resumen}

La fitorremediación es una tecnología alternativa de bajo costo utilizada para descontaminar suelos con metales pesados. En este ensayo se evaluó el uso de especies forestales de la sierra peruana (Acacia visco, Buddleja coriacea, Eucalyptus globulus, Myoporum laetum, Polylepis racemosa y Schinus molle) para el tratamiento de relaves mineros. Se evaluó el desarrollo de las especies plantadas en relave minero durante 27 semanas y se hizo un análisis fisicoquímico al relave para determinar el pH y la concentración de elementos químicos. Las especies forestales demostraron tener gran tolerancia a suelos contaminados y se obtuvo una remoción de los elementos químicos: antimonio (Sb), arsénico (As), cadmio (Cd), cobre (Cu), plata $(\mathrm{Ag})$ y plomo $(\mathrm{Pb})$.

Descriptores: relaves mineros, fitorremediación, contaminación, especies forestales.

\section{Abstract}

Phytoremediation is a low cost remediation technology used to decontaminate soils from heavy metals. In this study, the use of forest species from the Peruvian Andes (Acacia visco, Buddleja coriacea, Eucalyptus globulus, Myoporum laetum, Polylepis racemosa and Schinus molle) for the treatment of mine tailings, was evaluated. The development of the species in the mining waste was evaluated for 27 weeks, and the $\mathrm{pH}$ and concentration of chemical elements were analyzed. The employed species demonstrated a great grow tolerance in contaminated soils, and was removed chemical elements, such as antimony (Sb), arsenic (As), cadmium (Cd), copper $(\mathrm{Cu})$, silver $(\mathrm{Ag})$ and lead $(\mathrm{Pb})$.

Keywords: mining tailings, phytoremediation, contamination, forest species.

\section{Introducción}

La creciente contaminación de los suelos y del agua, derivada de la actividad industrial como la minería, plantea un desafío sobre cómo remediar las áreas contaminadas por la operación y funcionamiento de las mismas, que en su etapa de Cierre de Mina tienen una mayor dificultad para solucionar el problema de la concentración de contaminantes acumulados en depósitos de relaves, con potencial para dañar el medio ambiente y elevada capacidad de resistencia a la biodegradación [9].

La fitorremediación es una tecnología alternativa y sostenible; que consiste en el uso de plantas para reducir, degradar o inmovilizar compuestos orgánicos e inorgánicos considerados como contaminantes (naturales o sintéticos), del suelo, el agua o del aire. Se ha encontrado especies con la capacidad de almacenar metales pesados en alta concentración presentes en el suelo 
(hiperacumuladoras) y que resultan potencialmente tóxicos como U, Cd, Pb, Zn, $\mathrm{Cu}, \mathrm{Fe}, \mathrm{Ni}$, Se, etc. [2]

Existe estudios realizados por la Agencia de Protección Ambiental (EPA US) sobre la factibilidad de fitorremediación de metales pesados, habiendo cuatro mecanismos de absorción de metales en plantas: fitoextracción, fitovolatización, fitoestabilización y rizofiltración [3].

En México existe listados botánicos de especies arbóreas fitorremediadoras de suelos contaminados $y$, en algunos casos, en aguas contaminadas [10]. También hay especies para remover metales pesados, como el Sauce, Acacia, Mimosa, Anadenantera, Genipa americana y Eucalyptus; que han sido usados para la remoción de $\mathrm{Cd}, \mathrm{Pb}$ y $\mathrm{Cr}[11]$.

El objetivo de este trabajo es evaluar la aplicabilidad de especies forestales de la serranía peruana, para el tratamiento de fitorremediación en relaves mineros.

\section{Metodología}

\subsection{Caracterización del relave minero}

Se obtuvo una muestra representativa de aproximadamente media tonelada de relave minero proveniente de la relavera "Susana" de la "Cía. Minera Argentum S. A., Unidad Morocoha".

Se homogenizó el relave y se dejó secando al ambiente durante 48 horas. Aplicando el método del cuarteo, se seleccionó una porción de $1 \mathrm{~kg}$ de relave (muestra control de relave), llamada TOR2, de la cual se analizó el pH y el contenido de metales y metaloides. Este último se hizo por el método de ensayo fisicoquímico EPA 3050B [7] y estuvo a cargo de un laboratorio acreditado (ALS Corplab).

\subsection{Selección de especies para el ensayo}

Se consideraron especies forestales que crecen en la sierra del Perú, sobre los 2000 msnm. Este estrato ecológico está caracterizado por fuertes pendientes, un clima normalmente frío y, es donde la mayoría de industrias mineras se ubican, a lo largo de la cordillera de los Andes.

Se escogieron árboles de 6 meses de edad aproximadamente, con una altura similar.

\subsection{Disposición de los tratamientos}

El relave minero (RM) se colocó en cubetas de vidrio de $0.012 \mathrm{~m} 3$ de capacidad. Sobre el relave se puso tierra agrícola (TA), sin realizar una mezcla, de tal manera que ambos estratos puedan diferenciarse a simple vista.

Luego se sembró especímenes de cada especie vegetal seleccionada, agregando compost (100 $\mathrm{g} /$ planta) a la hora de sembrar las plantas para que se adapten más rápido a su nuevo sustrato.

Se dispuso 3 tratamientos de RM:TA (v:v) en las proporciones de 50:50 (T1R2); 80:20 (T2R2) y 100:0 (T3R2).

Los árboles fueron regados manteniendo la humedad del sustrato por un período de 27 semanas. Adicionalmente, se les aplicó biofertilizante (EM-1®, producto comercial) mezclado con el agua de riego en una proporción de $1 / 20$, para optimizar el desarrollo del organismo vegetal.

\subsection{Análisis del relave luego de los tratamientos}

Después de las 27 semanas los árboles fueron removidos de las cubetas y se tomaron muestras de $1.5 \mathrm{~kg}$ del RM para realizar los análisis de $\mathrm{pH}$ y contenido de metales y metaloides.

Esta operación se realizó con todas las cubetas, excepto con las del tratamiento T3R2, cuyos árboles fueron descartados por mortandad a los pocos días de haber iniciado la etapa experimental.

Se describe el procedimiento experimental y el equipamiento utilizado. Aquí no se muestran los resultados sino se describe la metodología empleada en el trabajo experimental.

\section{Resultados y discusión}

\subsection{Caracterización del relave minero}

Luego del análisis fisicoquímico practicado a la muestra T0R2, se constató la elevada concentración de metales pesados y metaloides contenidos en el relave (tabla 1), los cuales superan los ECAs (Estándares de Calidad Ambiental) para suelos (ECA-Suelo) a nivel nacional [6] e internacional [5] en sus tres categorías; para suelos agrícolas: el Sb supera en 15 veces, As en 44 veces, $C d$ en 26 veces, $C u$ en 87 veces, $\mathrm{Ag}$ en 3 veces y $\mathrm{Pb}$ en 42 veces. 
Además, el pH fue ácido (2.46), fuera de los ECA- Suelo (6 a 8).

Tabla 1: Resultados de la muestra TOR2 comparándola con los ECA-Suelo.

\begin{tabular}{lllll} 
& & \multicolumn{3}{c}{ ECA-Suelo (mg/kg) } \\
Parámetro & T0 R2 (mg/kg) & Agrícola & $\begin{array}{l}\text { Residencial/ } \\
\text { Parques }\end{array}$ & $\begin{array}{l}\text { Comercial/ } \\
\text { Industrial }\end{array}$ \\
\hline Antimonio (Sb) & $\mathbf{2 9 8 . 1}$ & $20^{(1)}$ & $20^{(1)}$ & $40^{(1)}$ \\
\hline Arsénico (As) & $\mathbf{2 2 0 2 . 0}$ & $50^{(2)}$ & $50^{(2)}$ & $140^{(2)}$ \\
\hline Cadmio (Cd) & $\mathbf{3 6 . 5 2}$ & $1.4^{(2)}$ & $10^{(2)}$ & $22^{(2)}$ \\
\hline Cobre (Cu) & $\mathbf{5 4 6 0 . 5}$ & $63^{(1)}$ & $63^{(1)}$ & $91^{(1)}$ \\
\hline Plata (Ag) & $\mathbf{6 1 . 6}$ & $20^{(1)}$ & $20^{(1)}$ & $40^{(1)}$ \\
\hline Plomo (Pb) & $\mathbf{2 9 3 3 . 5}$ & $70^{(2)}$ & $140^{(2)}$ & $1200^{(2)}$ \\
\hline pH & $\mathbf{2 . 5 0}$ & 6 a $^{(1)}$ & 6 a $^{(1)}$ & 6 a $^{(1)}$ \\
\hline
\end{tabular}

(1) Tomado de Canadian Soil Quality Guidelines for de protection of Enviromental and Human Health (CCME, 1996).

(2) Tomado del Decreto Supremo Nº02-2013-MINAM.

\subsection{Selección de especies para el ensayo}

La tecnología de fitorremediación ha sido ampliamente estudiada y se conocen varias especies de plantas con capacidad para "hiperacumular" selectivamente alguna sustancia; como la alfalfa, remolacha, col, tabaco, girasol, jamaica, mostaza india, entre otros [4, 12]. Pero, en su mayoría, se tratan de especies comestibles; lo cual significa un riesgo potencial por su consumo.

En el caso particular de esta investigación, los depósitos de relaves pueden alcanzar cientos de miles de metros cúbicos, lo que significa que el material contaminado abarca grandes volúmenes. Entonces, a gran escala la remoción de metales utilizando árboles puede ser más eficaz, principalmente debido al enraizamiento más profundo y un mayor rendimiento de biomasa [4].

Teniendo en consideración lo antes mencionado y, el estrato ecológico donde se ubica la mayoría de minas en el Perú, se seleccionó 6 especies forestales leñosas andinas: Vilco (Acacia visco), C'olle (Buddleja coriácea), Eucalipto (Eucalyptus globulus), Mioporo (Myoporum laetum), Quinual (Polylepis racemosa) y Molle (Schinus molle).

\subsection{Análisis del relave luego de los tratamientos}

Se analizó el pH y la concentración de los elementos químicos indicados antes, comparando los resultados de TOR2 con T1R2 y T2R2 para cada especie utilizada. Los resultados fueron expresados como el porcentaje de remoción (\%
Remoción) de elementos químicos analizados, para lo cual se empleó la siguiente fórmula:

$$
\% \text { Remoción }=\left(M R_{0}-M R_{f}\right) \times 100 / M R_{0}
$$

Dónde: MRO es la concentración del elemento químico en el relave T0R2 y, MRf es la concentración del elemento químico en el relave T1R2 o T2R2, según sea el caso.

Análisis del pH

Luego del tratamiento desarrollado se logró mejorar el pH ácido (2.46) del relave, llegando en la mayoría de casos a ser mayor a 6 (figura 1), dentro de los ECA-Suelo. Esto indicaría que los tratamientos mejoraron la calidad del sustrato, ya que hubo un aumento de materia orgánica a la vez que muchos de los metales eran removidos del medio. Es preciso mencionar que en los tratamientos con S. Molle el pH se conservó ácido, esto puede ser debido a que las raíces no abarcaron toda la zona donde se encontraba el relave.

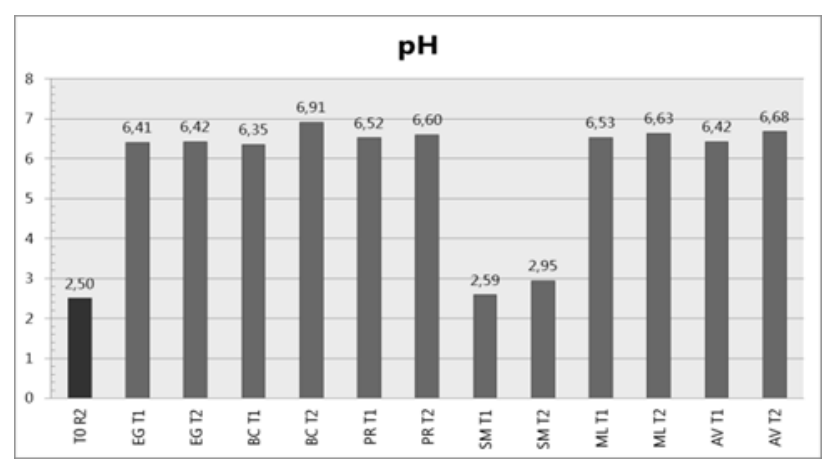


Figura 1: Registro del $\mathrm{pH}$ luego de los tratamientos. Remoción de elementos químicos

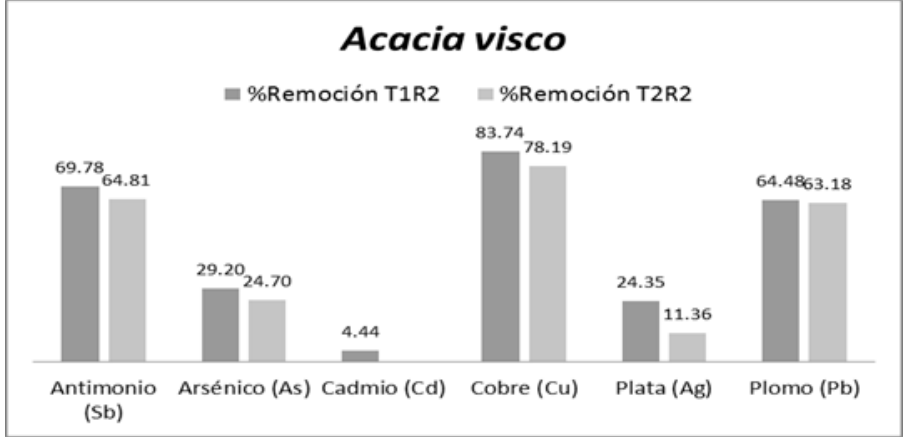

Figura 2: \%Remoción con Vilco (A. Visco).

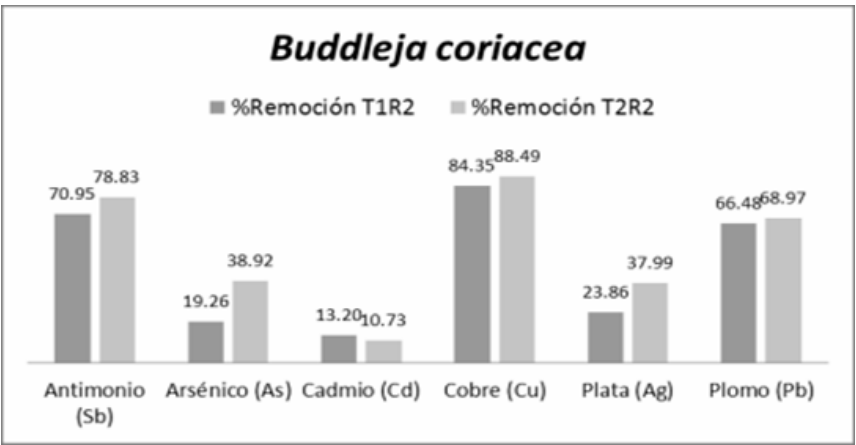

Figura 3: \%Remoción con C’olle (B. coriácea).

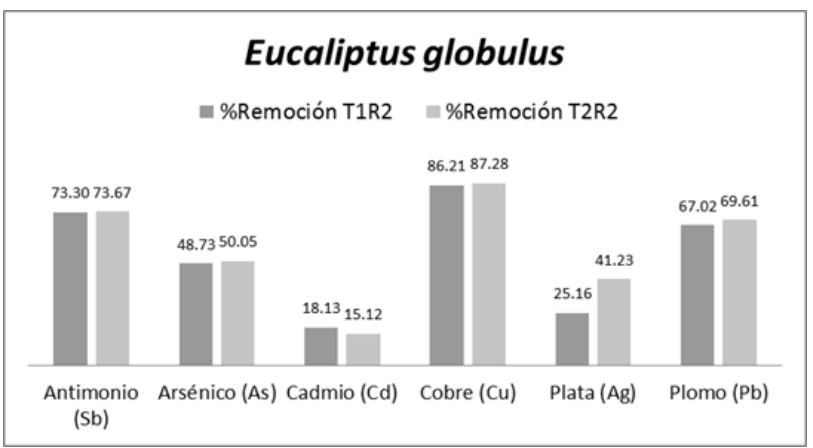

Figura 4: \%Remoción con Eucalipto (E. globulus).

Para los tratamientos T1R2 y T2R2, usando las especies A. visco, B. coriácea, E. globulus, M. laetum y $\mathrm{P}$. racemosa; se obtuvo un porcentaje de remoción de los elementos $\mathrm{Cu}, \mathrm{Sb}, \mathrm{Pb} \mathrm{As}, \mathrm{Ag}$ y $\mathrm{Cd}$. En el caso de S. molle, solo se logró obtener una remoción de $\mathrm{Cu}$, As y $\mathrm{Pb}$. En las figuras 2-7 se puede ver el detalle de los porcentajes de remoción (\% Remoción) obtenidos.

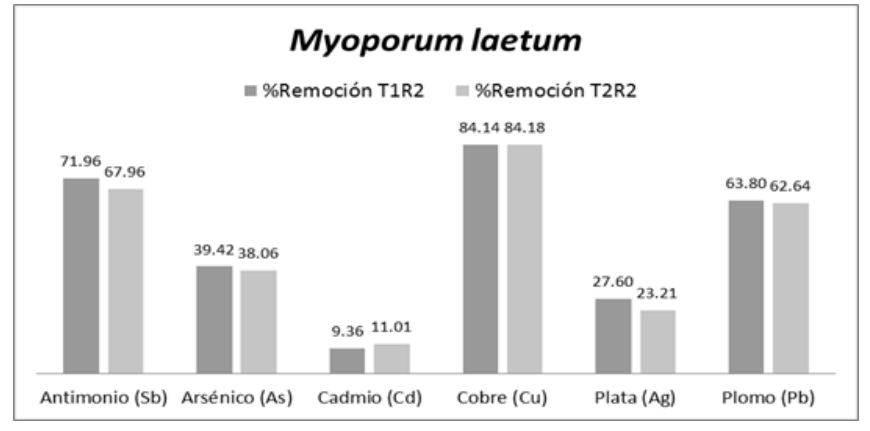

Figura 5: \%Remoción con Mioporo (M. Laetum)

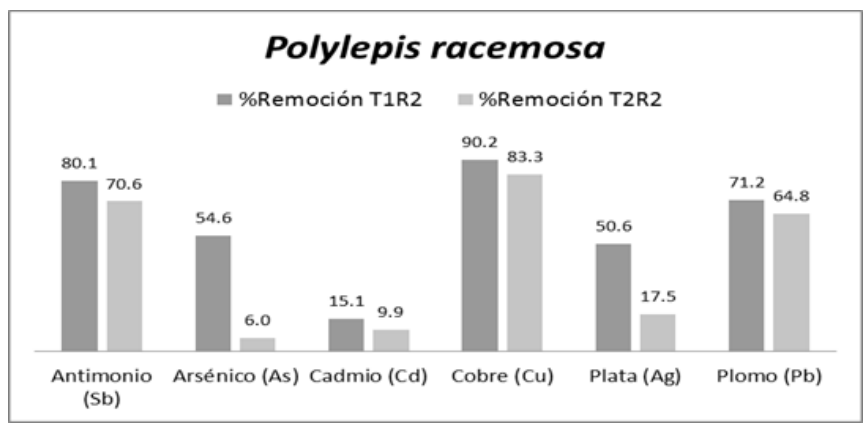

Figura 6: \%Remoción con Quinual (P. racemosa).

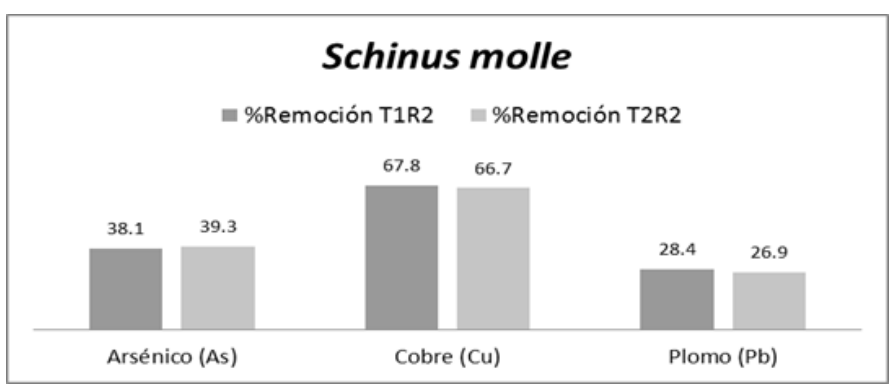

Figura 7: \%Remoción con Molle (S. molle).

Se pudo notar que hay una mayor remoción de $\mathrm{Cu}$ usando todas las especies evaluadas, siendo $P$. racemosa con la que se obtuvo el mayor porcentaje de remoción $(90.15 \%)$, esta especie también fue la que logró mayor remoción de $\mathrm{Sb}$ (80.14\%), As (54.62\%), Ag (50.65\%) y $\mathrm{Pb}$ (71.21\%). Para el Cd, el Eucalipto fue la especie con la que se obtuvo el mayor porcentaje de remoción (50.05\%).

Se ha encontrado referencias que muestran que la especie E. globulus es capaz de tolerar suelos contaminados con $\mathrm{Zn}$, $\mathrm{Cu}$ y $\mathrm{Pb}$ [4]; en ensayos de fitorremediación de contaminación por metales pesados, la especie E. camaldulensis es una de las más estudiadas. M. laetum tiene la capacidad de tolerar y acumular niveles tóxicos del metal $\mathrm{Pb}$; también indican que es capaz de desarrollarse en suelos contaminados con Zn [8]. 
De las demás especies usadas en este estudio, no se ha encontrado referencias donde se mencione su aplicación en fitorremediación.

Sería recomendable realizar el tratamiento del relave conformando un estrato de tierra agrícola (también llamado top soil), ya que esta capa de materia orgánica le proporcionará a la planta los nutrientes necesarios para que la planta no sufra un estrés excesivo al estar en contacto con el relave.

Las especies más grandes, como el Eucalipto o el Quinual, pueden ser utilizadas también en los depósitos de relaves o residuos mineros para mejorar la estabilidad física del terreno. Las especies de menor altura como el Mioporo o el C'olle se pueden colocar en el borde de las relaveras a modo de cerco vivo para delimitar el área.

\section{Conclusiones}

Los relaves mineros podrían ser tratados aplicando la técnica de fitorremediación con especies forestales que habitan en la sierra peruana. Las especies forestales seleccionadas demostraron tener la capacidad de tolerar suelos contaminados con relaves 0 metales pesados (especies metaltolerantes) y además tienen la capacidad de remediar los sitios contaminados, elevando el $\mathrm{pH}$ ácido y removiendo los elementos químicos del medio contaminado.

Se vierte las principales conclusiones derivadas del estudio. Las Conclusiones constituyen el punto principal para futuras investigaciones, comprende la interpretación de los resultados y las soluciones del problema estudiado.

\section{Agradecimientos}

A la empresa PROESMIN S.A.C., por apostar por la investigación en temas de suma importancia para el cuidado y remediación del medio ambiente, afectado por actividades mineras principalmente.

\section{Referencias}

[1] Arriagada et al., Improved zinc tolerance in Eucalyptus globulus inoculated with Glomus deserticola Trametes versicolor Corilopsis rigida. Soil Biology \& Biochemistry 42(2010). 118-124.

[2] P. Baldwin y D. Butcher, Phytoremediation of arsenic by two hyperaccumulatorsin a hydroponic environment. Microchemical Journal 85(2007)297-300. Elsevier B.V. EE.UU.

[3] C. Barid, Química ambiental, Reverté S.A. España, 2010.

[4] M. Capuana, Heavy metals and woody plantsbiotechnologies for

phytoremediation. Biogeosciences and Forestry 4(2011)7-15.

[5] Canadian Council of Ministers of the Environment (CCME), Canadian Soil Quality Guidelines for the Protection of Enviromental and Human Health, Canadá, 1996.

[6] Decreto Supremo No 002-2013-MINAM, Estándares de Calidad Ambiental (ECA) para Suelo,Perú, 2013.

[7] Enviromental Protection Agency (EPA). (1996). Method 3050B. Acid digestion of sediments, sludges, and soils. Revision 2. EE.UU.

[8] E. Lam \& I. Montofré, Phytoremediation of lead and zinc using Myoporum laetum and Brassica nigra in Antofagasta, Northern Chile, Advances In Geoecology, 40(2009)355-360. Land degradation and rehabilitation: dryland ecosystems. Alemania.

[9] M. López, Distribución y fitodisponibilidad de metales pesados ( $\mathrm{Sb}, \mathrm{Hg}, \mathrm{As}$ ) en los jales de la mina de antimonio de Wadley, estado de San Luis Potosí, Tesis para obtener el grado de Maestro en Ciencias de la Tierra. Universidad Autónoma de México, 2009.

[10] [1] G. Ochoa, S. Pérez, J.A. Frías, A. Jarquín y A. Méndez, Estudio prospectivo de especies arbóreas promisorias para la fitorremediación de suelos contaminados por hidrocarburos. Gobierno del Estado de Tabasco, Secretaría de Recursos Naturales y Protección Ambiental, El Colegio de la Frontera Sur y Petróleos Mexicanos, 2011, p 144.

[11] [1] A. C. C. Pereira, Heavy metals concentration in tree species used for revegetation of contaminated area, Revista Ciencia, Agronómica 43(4) (2012)641-647.

[12] B. Sepúlveda, O. Pavez y M. Tapia, Fitoextracción de metales pesados desde relaves utilizando plantas de Salicornia sp. Revista de la Facultad de Ingeniería, Universidad de Atacama. Chile 28 (2012)2026.

E-mail: jparedesmur.biotec@gmail.com 